\title{
Fine-Grained Recommendation Systems for Service Attribute Exchange
}

\author{
Christopher Staite ${ }^{1}$, Rami Bahsoon ${ }^{1}$, and Stephen Wolak ${ }^{2}$ \\ 1 School of Computer Science \\ University of Birmingham, United Kingdom \\ \{C.Staite, R.Bahsoon\}@ C. . bham.ac.uk \\ 2 Vodafone Group Plc, Newbury, United Kingdom \\ Stephen.Wolak@vodafone.com
}

\begin{abstract}
The effectiveness of service oriented computing relies on the trustworthiness of sharing of data between services. We advocate a semi-automated approach for information distribution and sharing, assisted by a reputation system. Unlike current recommendation systems which provide a user with a general trust value for a service, we propose a reputation model which calculates trust neighbourhoods through fine-grained multi-attribute analysis. Such a model allows a recommendation relevance to improve whilst maintaining a large user group, propagating and evolving trust perceptions between users. The approach is demonstrated on a small example.
\end{abstract}

\section{Introduction}

We address the problem of maintaining privacy where services interact with users. We suggest a recommendation system in order to assist sharing decisions and suggest semiautomation of sharing.

Current services maintain user profiles locally, which causes data to become outdated. The onus is on the user to provide a cross-service link between their profiles and manage authentication credentials. As a response, centralised Single Sign-On (SSO) services emerged permitting a single login. Popular implementations include Shibboleth [1] and OpenID [2]. This mechanism provides an easy framework for identifying users, but does not facilitate centralised profile storage.

Several systems attempt to centralise the storage of profile information. The most common is browser automatic form filling, which stores previous values. Although this assists profile creation, it does not allow services to access recent data. Other implementations such as SAML [3], OAuth [4] and OpenID Attribute Exchange [5] provide an interface to a central data repository. These permit centralised profile maintenance, assisting users on devices with restricted input abilities.

Sharing profile data between services raises many privacy questions, including: What trust should a user impart on a service? Which services should be allowed access to which parts of a users profile? How should data be transmitted between services? Where should the profile be stored? How can a user be assured that their data will not be used against the users' will? How can a service be sure that the user is not giving inaccurate information?

L. Baresi, C.-H. Chi, and J. Suzuki (Eds.): ICSOC-ServiceWave 2009, LNCS 5900, pp. 352_357, 2009.

(C) Springer-Verlag Berlin Heidelberg 2009 
As described in Section 2 research has attempted to solve the first question posed by using a recommendation system. Existing recommendation systems take coarse-grained approaches to analysis of trust by means of a single metric. Humans do not perceive trust on a per-service level, instead trust is dependant on factors such as the requested attributes. A user is constantly sharing their information as they utilise services, causing many requests from the central data. It is essential many of these operations are automated in order to produce a usable system. Services which have previously been given access could be given permanent access to certain fields. Initial access requests are hard to assess for trustworthiness from a mobile device. A recommendation from other users may provide a simple measure to assist.

We argue that there is a pragmatic need for semi-automated information distribution, assisted by reputation systems. By semi-automated we refer to the requirement of security to allow a user to make the decision in the case of sensitive data, but automatically distribute low-sensitivity data based on calculated trust. Reputation systems must analyse the requested attributes in order to accurately represent user opinion. In this paper we propose a mechanism, extending previous systems, in order to take attributes into account. Hence, we alter the analysis from the coarse-grained toward the fine-grained.

This paper is structured in the following manner: Section 2 discusses related work. Section 3 discusses the requirements for multi-factor trust analysis and proposes a formula which meets these requirements. A simple example is shown to detail the approach. Section 4 concludes.

\section{Related Work}

Current implementations of sharing mechanisms for the automation of attribute exchange (e.g. [6]7) require a considerable setup effort from the user, such as defining the context and purpose of use.

Existing service recommendations are based upon whole service reputation [8910]. [11] argues reputation systems are inadequate, due to the subtle differences between trust and reputation [12]. Trust defines a mental bias toward or against a service, whereas reputation is the conveyance of trust between people. The context sensitivity of trust causes it to be degraded by the use of coarse-grained reputation. We propose that reputation is more reliable when measured at a lower level (i.e. per-attribute).

[13] discusses methodologies for attribute exchange: although security in transmission is available, no mechanism utilises automation or recommendation.

[14] extends [13] in an attempts to enhance identity management online. Specifically, they provide users with the ability to see the purpose for request/retention details for each attribute, and the ability to revoke previously shared attributes [15]. This requires a large amount of screen space and bandwidth making its portability to mobile devices limited.

[1617] extend authentication systems to achieve privacy oriented attribute exchange by utilising security conditions (similar to SAML [3]) and oblivious transfer [18]. The computational complexity is significant and unsuitable for deployment on mobile devices. Further, these methods do not allow for a persistent profile among user devices.

Collaborative filtering produces recommendations between items based on previous user input in order to make recommendations (e.g. user-based [19] and item-based 
[20]). Both methods fail to apply multi-attribute to determine cross-attribute links (i.e. grouping users based on more than one attribute).

\section{Approach}

When a service requests personal information from a user it is assessed for trustworthiness. Abdul-Rahman \& Hailes [10] discuss the three types of trust imparted by the user. Interpersonal trust is the trust between two individuals in a specific context. This is the context in which the service is accessed and which attributes are requested. System trust defines the general trust level in an institution. This relates to the provider of the service. Dispositional trust defines the natural trust a user has in a third party before they take any other aspect into consideration. Many people are willing to share their personal information freely on the internet, others prefer to preserve their anonymity due to their disposition.

A recommendation system attempts to group users based on their dispositional trust. It may then inform a user of interpersonal and system trust through analysis of similar users' previous decisions. Previous implementations fail to properly assess interpersonal trust due to their coarse-grained analysis.

Dispositional trust may evolve over time, the proposed system does not consider this factor. Hence, the database of previous interactions should degrade over time. For performance reasons the groupings may be calculated offline. By iteratively calculating the groupings offline there is a constant and recent grouping matrix available for fast trust calculations.

The trust measurement is taken from the decision to share information with a service. We define a binary variable $P_{u, s, a}$, where $s$ identifies the service, $a$ identifies the attribute requested and $u$ identifies the user. The value is 0 if the information is withheld, or if a false value is given. If the attribute is supplied the value is defined as $1 . P_{u, s}$ refers to the set of attribute sharing values and may be in the state undefined if $u$ has no experience with $s$. The correlation between two users $(u 1, u 2)$ produces a recommendation. This is performed for all values of $P$ which are defined for both users $u 1$ and $u 2$. Where $P$ is the set of all $P_{u}$ for which $P_{u, s}$ are defined.

$$
\begin{gathered}
R_{u 1, s}=\frac{\sum_{i=2}^{|u|}\left(P_{u i, s} \operatorname{Cor}\left(P_{u 1}, P_{u i}\right)\right)}{\sum_{l=2}^{|u|} \operatorname{Cor}\left(P_{u 1}, P_{u l}\right)} \\
\operatorname{Cor}\left(P_{u 1}, P_{u i}\right)=\left|P_{u i}\right| \sum_{j=1}^{\left|P_{u i}\right|} 1-\sqrt{\frac{\sum_{k=1}^{\left|P_{u 1, s j}\right|}\left(P_{u 1, s j, a k}-P_{u i, s j, a k}\right)^{2}}{\left|P_{u 1, s j}\right|}}
\end{gathered}
$$

The correlation between two users is in a non-normalised form and used to provide the ratio of trust transference. This provides a non-negative value where 0 is defined as no similarities in past decisions, and increase in size the more similar previous decisions have been. A weighting value may be required in order to ignore lower correlation values. The multiplication by $\left|P_{u i}\right|$ ensures that users which have used more services similar to the user get preference over those who have similar, but limited, experience compared to the current user. The value of $R_{u 1, s}$ is a vector with sharing recommendations for each of the attributes which may be sent to $s$. Each of the values in the 


\begin{tabular}{|c|c|c|c|}
\hline \multirow{4}{*}{ Requested data } & Service 1 & Service 2 & Service 3 \\
\hline & $(0)$ & $(1)$ & $(1)$ \\
\hline & 1 & 0 & 1 \\
\hline & 1 & 1 & 0 \\
\hline & 0 & 1 & 1 \\
\hline \multirow[t]{2}{*}{ Alice } & 1 & 0 & 1 \\
\hline & 1 & 1 & 0 \\
\hline \multirow{3}{*}{$B o b$} & 0 & 1 & 1 \\
\hline & 0 & 0 & 0 \\
\hline & 1 & 1 & 0 \\
\hline Charlotte & 1 & $\begin{array}{l}1 \\
0\end{array}$ & undefined \\
\hline & $1)$ & $(1)$ & \\
\hline
\end{tabular}

Fig. 1. A scenario showing three users transactions with three services

vector are between 0 , meaning a strong recommendation not to share that attribute, and 1 which gives a strong recommendation to share the requested attribute. When the user has made their decision to share or withold attributes, the value of $P_{u, s, a}$ is set/altered.

We illustrate the use of the algorithm using a small example with the values given in Figure 1. In this table the requested attributes are shown for three services, followed by the previous sharing performed by three users, Alice, Bob and Charlotte. Both Bob and Alice have past transaction experience with all three services, Service 1, Service 2 and Service 3. Charlotte only has past experience with Service 1 and Service 2.

Charlotte visits Service 3 and an attribute request is placed from the service to gain data items 1 and 2. A decision about which attributes to provide must be made, the recommendation system will aid the service judgement based on previous experiences. In order to provide the recommendation we calculate $R_{\text {Charlotte,Service } 2 \text {. }}$

$$
\begin{gathered}
\operatorname{Cor}\left(P_{C, s 1}, P_{A, s 1}\right)=\sqrt{\frac{\sum_{k=1}^{\left|P_{C, s 1}\right|}\left(P_{C, s 1, a k}-P_{A, s 1, a k}\right)^{2}}{\left|P_{C, s 1}\right|}} \\
=\sqrt{\frac{(0-0)^{2}+(1-1)^{2}+(1-1)^{2}}{3}}=\sqrt{0}=0 . \\
\operatorname{Cor}\left(P_{C, s 2}, P_{A, s 2}\right)=\sqrt{\frac{\sum_{k=1}^{\left|P_{C, s 2}\right|}\left(P_{C, s 2, a k}-P_{A, s 2, a k}\right)^{2}}{\left|P_{C, s 2}\right|}}=0 . \\
\operatorname{Cor}\left(P_{C}, P_{A}\right)=2((1-0)+(1-0))=4 . \\
\operatorname{Cor}\left(P_{C}, P_{B}\right)=2\left(\left(1-\frac{1}{\sqrt{3}}\right)+(1-0)\right)=\frac{2}{\sqrt{3}} .
\end{gathered}
$$

The value of $R_{C, s 3}$ may be calculated using these correlations as groupings for users which Charlotte has similar sharing habits. The calculation that Alice has very similar habits to Charlotte as their correlation is much higher than that with Bob. 


$$
\begin{aligned}
R_{C, s 3} & =\frac{\sum_{i=2}^{|u|}\left(P_{u i, s 3} \operatorname{Cor}\left(P_{C}, P_{u i}\right)\right)}{\sum_{l=2}^{|u|} \operatorname{Cor}\left(P_{C}, P_{u l}\right)} \\
& =\frac{\sqrt{3}\left(\left(P_{A, s 3} \operatorname{Cor}\left(P_{C}, P_{A}\right)\right)+\left(P_{B, s 3} \operatorname{Cor}\left(P_{C}, P_{B}\right)\right)\right)}{4 \sqrt{3}+2} \\
& =\frac{\left(\left\{\begin{array}{l}
1 \\
1 \\
0
\end{array}\right\} \cdot 4 \sqrt{3}\right)+\left(\left\{\begin{array}{l}
1 \\
0 \\
0
\end{array}\right\} \cdot 2\right)}{4 \sqrt{3}+2} \\
& =\left\{\begin{array}{c}
1 \\
\frac{4 \sqrt{3}}{4 \sqrt{3}+2} \\
0
\end{array}\right\} .
\end{aligned}
$$

We can present this recommendation to Charlotte in a percentage or bar form. She is given a $100 \%$ recommendation to share attribute 1 , a $78 \%$ recommendation to share attribute 2 and a $0 \%$ recommendation to share attribute 3 . The recommendation for attribute 3 is omitted as it was never requested by the service. Once Charlotte has made her decision of which information to share with the service the value of $P_{C, s 3}$ is set for future reference by Charlotte and for producing recommendations for other users of the system.

Evolution of dispositional trust can occur as user opinion of a service becomes more or less trusting. The method given in this paper simply provides a method of calculating correlation based on past decisions. In order to allow for evolution of trust a time-based degradation may be performed before correlation calculation.

\section{Conclusions}

The need for automation and the role of recommendation systems have been outlined. Following, a mechanism to provide a recommendation has been proposed based on fine-grained past staring. The example provided an execution of the algorithm on a very small data set. A centrally accessible profile store is still required, following which an analysis of the effect of the proposed algorithm may be performed. The key to successful adoption of a serviced based web is the ability to exchange attributes without a large user effort.

\section{References}

1. Erdos, M., Cantor, S.: Shibboleth architecture draft v05 (2002), https://www.switch.ch/aai/docs/shibboleth/internet2/ draft-internet2-shibboleth-arch-v05.pdf

2. Recordon, D., Reed, D.: Openid 2.0: a platform for user-centric identity management. In: DIM 2006: Proceedings of the second ACM workshop on Digital identity management, pp. 11-16. ACM, New York (2006)

3. Cantor, S., Kemp, J., Philpott, R., Maler, E.: Assertions and protocols for the oasis security assertion markup language (saml) v2.0 (2005), http: / docs . oasis-open.org/ security/saml/v2.0/saml-core-2.0-os.pdf 
4. Atwood, M., Conlan, R.M., Cook, B., Culver, L., Elliott-McCrea, K., Halff, L., HammerLahav, E., Laurie, B., Messina, C., Panzer, J., Quigley, S., Recordon, D., Sandler, E., Sergent, J., Sieling, T., Slesinsky, B., Smith, A.: OAuth Core 1.0. Technical report (2007)

5. Hardt, D., Bufu, J., Hoyt, J.: Openid atrribute exchange 1.0 - final (2007), http://openid.net/specs/openid-attribute-exchange-1_0.html

6. Cheng, W., Li, J., Moore, K., Karp, A.H.: A customer-centric privacy protection framework for mobile service-oriented architectures. In: IEEE International Conference on Services Computing, SCC 2008, Honolulu, HI, vol. 2, pp. 13-20 (2008)

7. Hong, J.I., Landay, J.A.: An architecture for privacy-sensitive ubiquitous computing. In: MobiSys 2004: Proceedings of the 2nd international conference on Mobile systems, applications, and services, pp. 177-189. ACM, New York (2004)

8. Herlocker, J.L., Konstan, J.A., Borchers, A., Riedl, J.: An algorithmic framework for performing collaborative filtering. In: SIGIR 1999: Proceedings of the 22nd annual international ACM SIGIR conference on Research and development in information retrieval, pp. 230-237. ACM, New York (1999)

9. Kinateder, M., Rothermel, K.: Architecture and algorithms for a distributed reputation system. In: Nixon, P., Terzis, S. (eds.) iTrust 2003. LNCS, vol. 2692, pp. 1-16. Springer, Heidelberg (2003)

10. Abdul-Rahman, A., Hailes, S.: Supporting trust in virtual communities, vol. 1, p. 9 (2000)

11. Resnick, P., Kuwabara, K., Zeckhauser, R., Friedman, E.: Reputation systems. Commun. ACM 43, 45-48 (2000)

12. Jøsang, A., Ismail, R., Boyd, C.: A survey of trust and reputation systems for online service provision. Decision Support Systems 43, 618-644 (2007) Emerging Issues in Collaborative Commerce

13. Pfitzmann, B., Waidner, M.: Privacy in browser-based attribute exchange. In: WPES 2002: Proceedings of the 2002 ACM workshop on Privacy in the Electronic Society, pp. 52-62. ACM, New York (2002)

14. Camenisch, J., Shelat, A., Sommer, D., Fischer-Hübner, S., Hansen, M., Krasemann, H., Lacoste, G., Leenes, R., Tseng, J.: Privacy and identity management for everyone. In: DIM 2005: Proceedings of the 2005 workshop on Digital identity management, pp. 20-27. ACM, New York (2005)

15. Pettersson, J.S., Fischer-Hübner, S., Danielsson, N., Nilsson, J., Bergmann, M., Clauss, S., Kriegelstein, T., Krasemann, H.: Making PRIME usable. In: Proceedings of the 2005 symposium on Usable privacy and security, pp. 53-64. ACM, New York (2005)

16. Fujiwara, S., Komura, T., Okabe, Y.: A privacy oriented extension of attribute exchange in shibboleth. In: IEEE/IPSJ International Symposium on Applications and the Internet Workshops, p. 28 (2007)

17. Takagi, T., Komura, T., Miyazaki, S., Okabe, Y.: Privacy oriented attribute exchange in shibboleth using magic protocols, pp. 293-296 (2008)

18. Rabin, M.: How to exchange secrets by oblivious transfer. Technical report, Technical Report TR-81, Harvard Aiken Computation Laboratory (1981)

19. Resnick, P., Iacovou, N., Suchak, M., Bergstrom, P., Riedl, J.: Grouplens: an open architecture for collaborative filtering of netnews. In: CSCW 1994: Proceedings of the 1994 ACM conference on Computer supported cooperative work, pp. 175-186. ACM, New York (1994)

20. Sarwar, B., Karypis, G., Konstan, J., Reidl, J.: Item-based collaborative filtering recommendation algorithms. In: WWW 2001: Proceedings of the 10th international conference on World Wide Web, pp. 285-295. ACM, New York (2001) 